\title{
Clinical and Technical Considerations for Brain PET Imaging for Dementia
}

Shelley N. Acuff ${ }^{1}$, Sulantha Mathotaarachchi ${ }^{2}$, Katherine Zukotynski ${ }^{3}$, Dustin Osborne ${ }^{1}$, and Rathan Subramaniam ${ }^{4}$, for the Alzheimer's Disease Neuroimaging Initiative

\begin{abstract}
${ }^{I}$ Department of Radiology, University of Tennessee Medical Center, University of Tennessee Graduate School of Medicine, Knoxville, Tennessee; ${ }^{2}$ McGill University Research Center for Studies in Aging, Montreal, Quebec, Canada; ${ }^{3}$ Departments of Medicine and Radiology, McMaster University, Hamilton, Ontario, Canada; and ${ }^{4}$ Division of Nuclear Medicine, Department of Radiology, Harold Simmons Comprehensive Cancer Center, University of Texas Southwestern Medical Center, Dallas, Texas
\end{abstract}

CE credit: For CE credit, you can access the test for this article, as well as additional JNMT CE tests, online at https://www.snmmilearningcenter.org. Complete the test online no later than March 2023. Your online test will be scored immediately. You may make 3 attempts to pass the test and must answer $80 \%$ of the questions correctly to receive $1.0 \mathrm{CEH}$ (Continuing Education Hour) credit. SNMMI members will have their CEH credit added to their VOICE transcript automatically; nonmembers will be able to print out a CE certificate upon successfully completing the test. The online test is free to SNMMI members; nonmembers must pay $\$ 15.00$ by credit card when logging onto the website to take the test.

The number of cases of dementia has dramatically increased over the last decade. Imaging of the brain with PET has been used for many years, but in the past decade the radiopharmaceuticals and technology available for imaging dementia have vastly improved. In recent years, the U.S. Food and Drug Administration has approved 3 PET radiopharmaceuticals for detecting amyloid in brain, and tau PET radiopharmaceuticals are being investigated in clinical trials for use in dementia imaging. This paper will discuss different forms of dementia that can be imaged with PET, review common radiopharmaceuticals used for imaging dementia, and provide technical recommendations for performing the studies.

Key Words: brain; PET; amyloid; tau

J Nucl Med Technol 2020; 48:5-8

DOI: $10.2967 /$ jnmt.118.220087

\section{$\mathbf{P}$}

ET has been in use for many years to evaluate patients with a host of different types of disease, including oncologic, cardiac, and neurologic etiologies (1). In recent years, PET has vastly improved through technologic developments and an increase in the number of PET radiopharmaceuticals available for imaging. Further, through the combination of PET with CT or MRI, the dream of being able to examine cell metabolism or to target specific proteins in a known anatomic location has become a reality. Although CT is not required for PET brain imaging, PET/CT is the preferred method for scanning brains. For years, the most widely used

\footnotetext{
Received Apr. 29, 2019; revision accepted May 13, 2019.

For correspondence or reprints contact: Shelley N. Acuff, University of Tennessee Graduate School of Medicine, 1924 Alcoa Hwy., Knoxville, TN 37920.

E-mail: sacuff@utmck.edu

Published online Jun. 10, 2019.

COPYRIGHT (c) 2020 by the Society of Nuclear Medicine and Molecular Imaging.
}

radiopharmaceutical for brain imaging was ${ }^{18} \mathrm{~F}-\mathrm{FDG}(1)$. ${ }^{18} \mathrm{~F}-\mathrm{FDG}$ is still commonly used in clinical practice to determine cerebral glucose metabolism, and the spatial pattern of ${ }^{18} \mathrm{~F}-\mathrm{FDG}$ metabolism is valuable in differentiating types of dementias. Alzheimer disease (AD) is a common form of dementia characterized by the accumulation of $\beta$-amyloid protein or senile plaque formation and neurofibrillary tangles of hyperphosphorylated tau protein (2). Today, several radiopharmaceuticals targeting amyloid protein are available, and specific radiopharmaceuticals targeting tau protein are in development. In this paper, we discuss more readily available PET radiopharmaceuticals for imaging dementia, the guidelines for their administration, and scanning techniques.

\section{TYPES OF BRAIN IMAGING}

In recent years, several PET radiopharmaceuticals have been developed for imaging subjects with mild cognitive impairment or dementia. Broadly, the goal of this imaging falls into 3 categories: detection of cerebral glucose metabolism, detection of cerebral amyloid protein, and detection of cerebral tau protein. Often preceded by mild cognitive impairment, dementia is defined as a group of neurodegenerative disorders characterized by memory impairment and cognitive decline resulting in difficulty in performing the activities of daily living for a minimum of $6 \mathrm{mo}(2,3)$. There are several types of dementia, typically categorized according to the results of a physical examination and neurocognitive tests, with imaging used to support the diagnosis. The most common types of dementia encountered in the nuclear medicine clinic are $\mathrm{AD}$, Lewy body dementia, frontotemporal dementia, and vascular dementia (2). AD is thought to account for up to $60 \%-80 \%$ of all dementia cases $(2,4)$.

\section{Cerebral Glucose Metabolism}

The brain metabolizes glucose, and ${ }^{18} \mathrm{~F}-\mathrm{FDG}$ is a glucose analog. The cerebral metabolic rate is an indicator of normal 
versus declining brain function. In patients with cognitive impairment, detection of decreased cerebral metabolic rate can be valuable, and an ${ }^{18}$ F-FDG scan may allow members of the health-care team to follow and manage patients as they progress from mild cognitive impairment to dementia (2). ${ }^{18}$ F-FDG scanning plays an important role in all brain imaging applications and serves as a valuable marker to differentiate $\mathrm{AD}$ from other causes of dementia, such as frontotemporal dementia, because an ${ }^{18} \mathrm{~F}$-FDG scan can indicate the brain regions with a decreased cerebral metabolic rate (Fig. 1) (5).

\section{Amyloid Protein Imaging}

It has been suggested that deposition of amyloid protein in the brain ( $\beta$-amyloid accumulation) is a slow process and may extend for decades before clinical symptoms are detected (4). Currently, investigational drugs to treat AD are focused mainly on arresting the progression of amyloid protein deposition, which is why it is so important to have a way to image $\beta$-amyloid accumulation over time. Over the last few decades, several radiopharmaceuticals have been developed with the ability to detect amyloid in the brain. The first was ${ }^{11} \mathrm{C}$-Pittsburgh compound $\mathrm{B}$. The biggest limitation for this radiopharmaceuticals was the short half-life of ${ }^{11} \mathrm{C}(20 \mathrm{~min})$ and the need for an onsite cyclotron (6). More recently, 3 radiopharmaceuticals that use ${ }^{18} \mathrm{~F}$ as the radioactive label have been developed (half-life of $110 \mathrm{~min}$ ) and have received Food and Drug Administration approval. The first to receive approval was ${ }^{18} \mathrm{~F}$-florbetapir (Amyvid; Eli Lilly), the next was ${ }^{18}$ F-flutemetamol (Vizamyl; GE Healthcare), and the last was ${ }^{18}$ F-florbetaben (Neuraceq; Piramal). It is important to remember, with these imaging studies, that just because radiopharmaceutical uptake may be observed in the brain does not mean the patient has AD. However, negative findings on an amyloid scan suggest a low likelihood that the cognitive impairment is caused by AD (Fig. 1) (2). With any PET radioligand, off-target uptake may be observed. Thus, the reader must be adequately trained in the interpretation of images from these specific compounds.

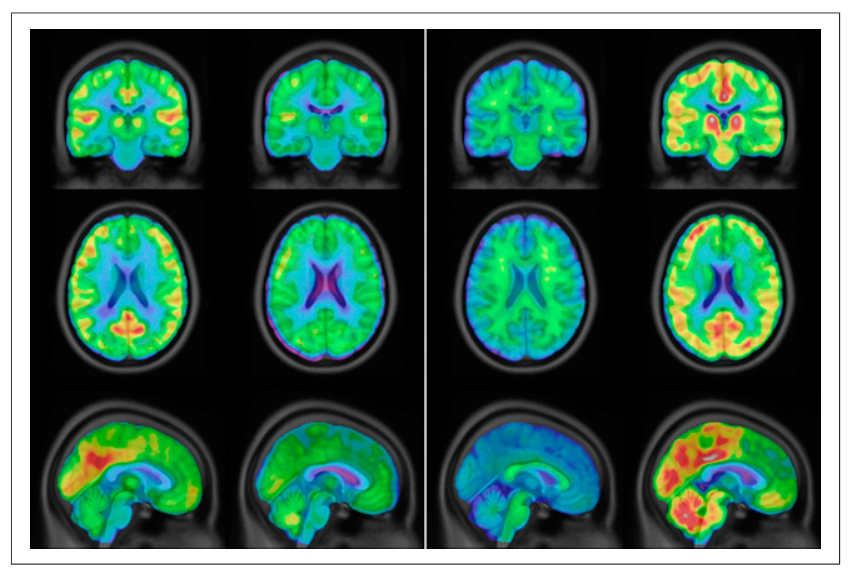

FIGURE 1. Normal (column 1) and abnormal (column 2) ${ }^{18} \mathrm{~F}-$ FDG PET findings and normal (column 3) and abnormal (column 4) ${ }^{18}$ F-florbetapir PET findings.

\section{Tau Protein Imaging}

The presence of neurofibrillary tangles of hyperphosphorylated tau proteins is also a hallmark of $\mathrm{AD}(4)$. In the healthy brain, tau proteins should bind to microtubules inside neurons to help support those cells and their functions; however, in $\mathrm{AD}$, changes to the chemistry of the brain can result in tau protein aggregation rather than binding to the microtubules. Over time, these aggregates form neurofibrillary tangles that disrupt communication between neurons (7). More recent work suggests that these tau tangles may disrupt communication between the nucleus and the body of neurons in the brain, adding to possible communication disruption mechanisms and further exacerbating neurodegeneration (8). Imaging with tau PET agents has shown a correlation between the severity of dementia and tau pathology (9).

Both amyloid and tau imaging agents have corroborated well with the Braak and Braak stages of amyloid protein and tau protein deposition of $\mathrm{AD}$, starting from the medial temporal regions in the early stages of the disease and moving to more widespread neocortical accumulation in the later stages (10). Currently, several tau PET radiopharmaceuticals are being studied in clinical trials, but none has been approved by the Food and Drug Administration or is being used routinely in clinical practice.

\section{BRAIN IMAGING GUIDELINES}

\section{Patient Preparation}

Patient preparation before a brain PET scan for clinically suspected dementia should start with a detailed conversation with the patient and family members. Issues to be discussed include patient history, symptoms, patient or physician concerns, and instructions on how the scanning will be performed. Through this discussion, the technologist will be able to assess the brain function of the patient and have an idea of how well the patient can cooperate during imaging. This also gives family members a chance to express their concerns and raise questions. Depending on the type of imaging ordered, specific guidelines may need to be followed for patient preparation.

For an ${ }^{18} \mathrm{~F}-\mathrm{FDG}$ brain PET/CT scan, the patient's environment before scanning is important. The patient needs to be in a quiet room with low ambient light and few stimuli for at least $30 \mathrm{~min}$ before radiopharmaceutical injection and throughout the 30- to 60-min uptake time. An intravenous line needs to be placed at least $10 \mathrm{~min}$ before the radiopharmaceutical injection, and a blood glucose test should be performed (i.e., a blood glucose level under $200 \mathrm{mg} / \mathrm{dL}$ is recommended before injection of the radiopharmaceutical). The patient must have had nothing to eat or drink for at least $4 \mathrm{~h}$ before the study (other than water and daily medication taken with water) (11).

When brain PET/CT is done using amyloid radiopharmaceuticals, little patient preparation is needed. An intravenous line should be established and the patient made comfortable. Environmental stimulation is not a concern, and patients may move freely in the uptake room as desired. 
They may also read or watch television. There are no food restrictions, and all daily medication may be taken.

\section{Injection of Radiopharmaceuticals and Uptake Time Guidelines}

After an intravenous line is in place, the shortest intravenous extension line available should be used when injecting the radiopharmaceuticals. The radiopharmaceutical should not be injected if any particulate matter is present. All radiopharmaceuticals should be injected as a slow bolus (within $40 \mathrm{~s}$ ) followed by a 10 - to $20-\mathrm{mL}$ saline flush of the intravenous line. Of note, unlike ${ }^{18} \mathrm{~F}-\mathrm{FDG}$, radiopharmaceuticals targeting amyloid do not allow for volume adjustment or flushing of the radiopharmaceutical syringe with saline. Details on the activity to administer and the uptake time are presented in Table 1. Once the injection is complete, the intravenous line can be removed.

\section{Patient Positioning}

At the end of the uptake time, the patient is asked to void. The mental status of the patient should be kept in mind and plenty of time allowed for use of the restroom and positioning of the patient on the scanner. The PET scan should be started on time to optimize the workflow in the PET center.

Proper head positioning for brain imaging is truly important. Before patients are positioned on the table, all items should be removed from their pockets, jewelry should be removed from the neck up, and hearing aids should be removed. The appropriate radiolucent head holder should be installed, as mild rotation or movement of the head can interfere with image interpretation. The patient is positioned supine on the table with the arms cross over the chest. The vertex of the head should reach the most superior point of the head holder. A foam wedge is used under the knees for lower back support, an arm strap to help stabilize the arms, and any other accessories needed to make the patient as comfortable as possible. Taking the time to complete these steps is essential to reduce body movement during the scan.

The laser lights on the PET/CT scanner are used to carefully position the head such that there is no rotation. The canthomeatal line should be oriented in the vertical position. Once the head is in the desired position, rolled washcloths or foam wedges are used to fill any gaps between the patient's head and the head holder. A head strap is secured across the forehead and the positioning rechecked with the laser lights (Fig. 2). The whole brain and cerebellum need

TABLE 1

Administered Activity and Uptake Time

\begin{tabular}{|c|c|c|}
\hline Tracer & $\begin{array}{c}\text { Activity } \\
\text { administered } \pm 10 \% \\
(\mathrm{MBq})\end{array}$ & $\begin{array}{c}\text { Uptake } \\
\text { time (min) }\end{array}$ \\
\hline${ }^{18} \mathrm{~F}-\mathrm{FDG}$ & 370 & $30-60$ \\
\hline${ }^{18}$ F-florbetapir (Amyvid) & 370 & $30-50$ \\
\hline${ }^{18}$ F-flutemetamol (Vizamyl) & 185 & $60-120$ \\
\hline${ }^{18} \mathrm{~F}$-florbetaben (Neuraceq) & 299.7 & $45-130$ \\
\hline
\end{tabular}

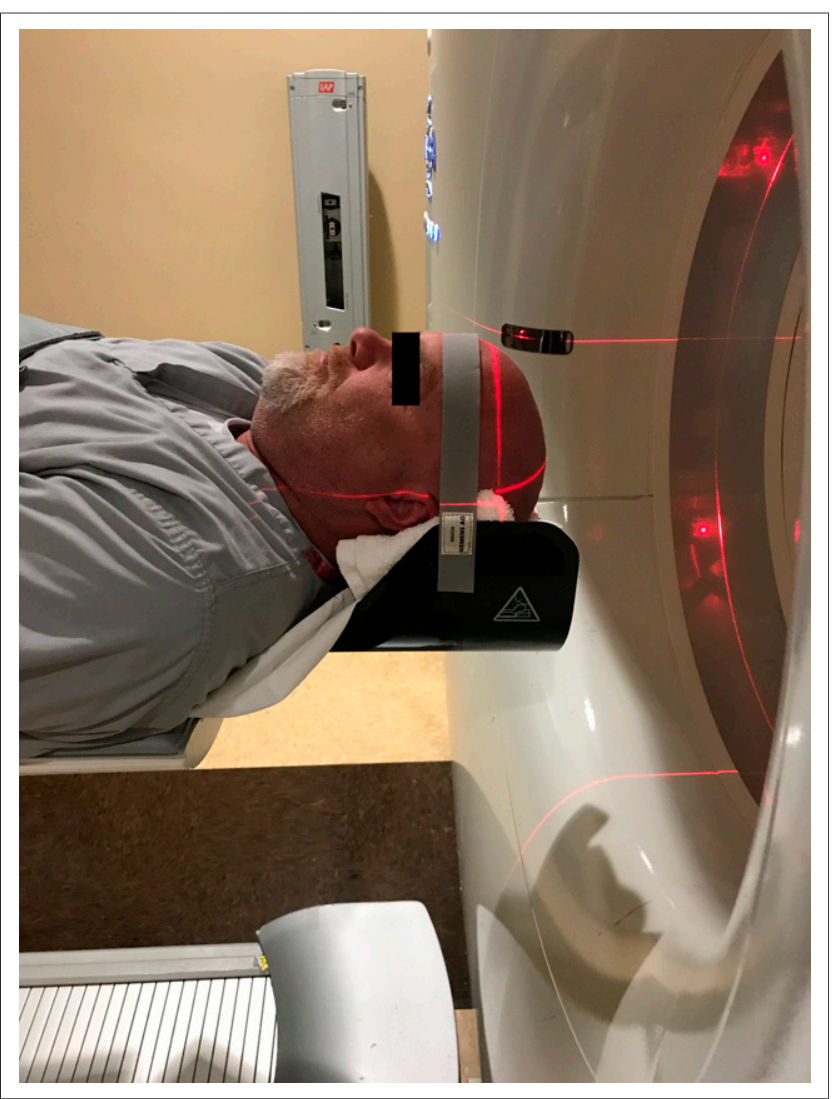

FIGURE 2. Proper head positioning using radiolucent holder with stabilizing accessories.

to be positioned in the field of view. Finally, the vertical placement of the table is checked to ensure that the brain is centrally located in the field of view. The patient should be given clear, detailed instructions on what to expect and the importance of remaining as still as possible.

\section{Imaging}

Depending on the imaging equipment available and the clinical indication, a single-field-of-view static image or a multiple sequential images of the same field of view, known as dynamic imaging, are performed. Static imaging is useful when the SUV is of interest. To calculate SUV, the technologist must document the patient's accurate weight, height, and the actual amount of radiopharmaceutical that was injected into the patient. Dynamic imaging is useful

TABLE 2

Scan Duration for Each Radiopharmaceutical

\begin{tabular}{lcc}
\hline \multicolumn{1}{c}{ Tracer } & $\begin{array}{c}\text { Static } \\
\text { image (min) }\end{array}$ & $\begin{array}{c}\text { Static sequence } \\
\text { imaging } \\
\text { (frames } \times \text { min) }\end{array}$ \\
\hline${ }^{18}$ F-FDG & 15 & $3 \times 5$ \\
${ }^{18}$ F-florbetapir (Amyvid) & 10 & $2 \times 5$ \\
${ }^{18}$ F-flutemetamol (Vizamyl) & $10-20$ & $2 \times 5$ \\
${ }^{18}$ F-florbetaben (Neuraceq) & $15-20$ & $4 \times 5$ \\
\hline
\end{tabular}


when quantification of the regional metabolic rate is of interest (11). When dynamic imaging is available, it is the preferred method; however, it is generally used in research settings because the scan can take up to $180 \mathrm{~min}$, with imaging starting at the time of injection. PET brain images typically are reconstructed into a $128 \times 128$ or $256 \times 256$ matrix. Iterative reconstruction is routinely used. Other reconstruction settings can be set according to institutional and camera manufacturing guidelines. Table 2 presents scan durations for each radiopharmaceutical. Often, multiple acquisitions are obtained during the scan. For example, 2 static images of the brain lasting 5 min each are typically acquired for each ${ }^{18} \mathrm{~F}$-florbetapir scan.

\section{CONCLUSION}

Although the underlying cause of dementia remains an enigma and a cure is still beyond our grasp, there has been much research over the last decade to improve our knowledge about the disease, how it is diagnosed, and how it is treated. PET of the brain is a helpful tool for confirming the diagnosis and assessing disease progression. In recent years, several new radiopharmaceuticals have become available for dementia imaging. With these new radiopharmaceuticals come new guidelines and recommendations to follow to obtain the best possible results for our patients.

\section{DISCLOSURE}

Data collection and sharing for this project were funded by the Alzheimer's Disease Neuroimaging Initiative (ADNI) (National Institutes of Health grant U01 AG024904) and the Department of Defense ADNI (Department of Defense award W81XWH-12-2-0012). ADNI is funded by the National Institute on Aging, the National Institute of Biomedical Imaging and Bioengineering, and generous contributions from the following: AbbVie; Alzheimer's Association; Alzheimer's Drug Discovery Foundation; Araclon Biotech; BioClinica, Inc.; Biogen; Bristol-Myers Squibb Company; CereSpir, Inc.; Cogstate; Eisai Inc.; Elan Pharmaceuticals, Inc.; Eli Lilly and Company; EuroImmun; F. Hoffmann-La Roche Ltd. and its affiliated company Genentech, Inc.; Fujirebio; GE Healthcare; IXICO Ltd.; Janssen Alzheimer Immunotherapy Research \& Development, LLC; Johnson \& Johnson Pharmaceutical Research \& Development LLC; Lumosity; Lundbeck; Merck \& Co., Inc.; Meso Scale Diagnostics, LLC; NeuroRx Research; Neurotrack Technologies; Novartis Pharmaceuticals Corporation; Pfizer Inc.; Piramal Imaging; Servier; Takeda Pharmaceutical Company; and Transition Therapeutics. The Canadian
Institutes of Health Research provide funds to support ADNI clinical sites in Canada. Private-sector contributions are facilitated by the Foundation for the National Institutes of Health (www.fnih.org). The grantee organization is the Northern California Institute for Research and Education, and the study is coordinated by the Alzheimer's Therapeutic Research Institute at the University of Southern California. ADNI data are disseminated by the Laboratory for Neuro Imaging at the University of Southern California. No other potential conflict of interest relevant to this article was reported.

\section{ACKNOWLEDGMENTS}

Data used in preparation of this article were obtained from the ADNI database (http://adni.loni.usc.edu/). As such, the investigators within the ADNI contributed to the design and implementation of ADNI or provided data but did not participate in analysis or writing of this report. A complete listing of ADNI investigators can be found at http://adni.loni.usc. edu/wp-content/uploads/how_to_apply/ADNI_Acknowledgement_ List.pdf.

\section{REFERENCES}

1. Portnow LH, Vaillancourt DE, Okun MS. The history of cerebral PET scanning: from physiology to cutting-edge technology. Neurology. 2013;80:952-956.

2. Marcus C, Mena E, Subramaniam RM. Brain PET in the diagnosis of Alzheimer's disease. Clin Nucl Med. 2014;39:e413-e422.

3. Eschweiler GW, Leyhe T, Kloppel S, Hull M. New developments in the diagnosis of dementia. Dtsch Arztebl Int. 2010;107:677-683.

4. Villemagne VL, Dore V, Bourgeat P, et al. Abeta-amyloid and tau imaging in dementia. Semin Nucl Med. 2017;47:75-88.

5. Jeong Y, Cho SS, Park JM, et al. ${ }^{18}$ F-FDG PET findings in frontotemporal dementia: an SPM analysis of 29 patients. J Nucl Med. 2005;46:233-239.

6. Johnson KA, Minoshima S, Bohnen NI, et al. Appropriate use criteria for amyloid PET: a report of the Amyloid Imaging Task Force, the Society of Nuclear Medicine and Molecular Imaging, and the Alzheimer's Association. Alzheimers Dement. 2013;9:e-1-e-16.

7. Bancher C, Brunner C, Lassmann H, et al. Accumulation of abnormally phosphorylated tau precedes the formation of neurofibrillary tangles in Alzheimer's disease. Brain Res. 1989;477:90-99.

8. Eftekharzadeh B, Daigle JG, Kapinos LE, et al. Tau protein disrupts nucleocytoplasmic transport in Alzheimer's disease. Neuron. 2019;101:349.

9. Villemagne VL, Dore V, Burnham SC, Masters CL, Rowe CC. Imaging tau and amyloid-beta proteinopathies in Alzheimer disease and other conditions. Nat Rev Neurol. 2018;14:225-236.

10. Braak H, Braak E. Neuropathological stageing of Alzheimer-related changes. Acta Neuropathol (Berl). 1991;82:239-259.

11. Society of Nuclear Medicine procedure guidelines for FDG PET brain imaging. SNMMI website. http://snmmi.files.cms-plus.com/docs/PET_COE/SNM\%20Procedure $\%$ 20Guideline\%20for\%20FDG\%20PET\%20Brain\%20Imaging.pdf. Published February 8, 2009. Accessed August 15, 2019. 